\title{
Validating New Technologies to Treat Depression, Pain and the Feeling of Sentient Beings: A Reply to "Neuroscience for the Soul"
}

\author{
Michael A. Persinger ${ }^{1,2,3^{*}}$, Todd R. Murphy ${ }^{1}$ \\ ${ }^{1}$ Behavioural Neuroscience Program, Laurentian University, Sudbury, Canada \\ ${ }^{2}$ Biomolecular Sciences Program, Laurentian University, Sudbury, Canada \\ ${ }^{3}$ Human Studies Program, Laurentian University, Sudbury, Canada \\ Email: ”mpersinger@laurentian.ca,drpersinger@neurocog.ca, brainsci@.j.net
}

Received 6 March 2016; accepted 26 March 2016; published 29 March 2016

Copyright (C) 2016 by authors and Scientific Research Publishing Inc.

This work is licensed under the Creative Commons Attribution International License (CC BY). http://creativecommons.org/licenses/by/4.0/

(c) (i) Open Access

\section{Abstract}

The primary assumption of Neuroscience is that all experiences are strongly correlated with or caused by the specifics of brain structures and their particular dynamics. The profound experiences attributed to the "sensed presence" and their cultural anthropomorphisms such as deities and gods are persistent reports in human populations that are frequently associated with permanent changes in behavior, reduced depression and alleviation of pain. The majority of traditional clinical observations and modern imaging techniques have emphasized the central role of right temporal lobe structures and their directly related networks. The experimental simulation of sensed presences which can result in attributions to spiritual, deity-based or mystical sources within the clinical laboratory by the application of physiologically-patterned magnetic fields across the temporal lobes through our God Helmet requires the same precision of technology that is essential for synthesizing molecular treatments for modifying anomalous behavior, depression and pain. Despite the clinical utility of these simulated conditions within Neuroscience and Medicine, misinformation concerning the bases and efficacy of this new technology persist. Here we present detailed technical clarifications and rebuttals to refute these misconceptions. A Hegelian approach to this delay of development and impedance provides a context through which the ultimate synthesis and application of this technology may be accommodated in the near future.

\section{Keywords}

Sensed Presence, Physiologically Patterned Magnetic Fields, Temporal Lobes, Neurotheology,

\footnotetext{
${ }^{*}$ Corresponding author.
} 


\section{Introduction}

Hegel's observation that a novel discovery or idea, a thesis, is followed by an antithesis that then results in a synthesis has been conspicuous within the progress of human knowledge [1]. There is balance when new knowledge appears within the traditional aggregate of assumptions but only if the antithesis is as valid as the thesis. In the history of Medicine and Neuroscience, there have been multiple examples where a new and valid idea is delayed or suppressed from development through systematic replication by the general scientific community due to persistently vocal misrepresentations and fallacious arguments by antagonists whose interests are based upon agendas or maintenance of the status quo rather than data-based criteria. As a result, the potential benefits from these new discoveries for the human population have been delayed or denied. The most recent perturbation concerns the validity and efficacy of specific temporally patterned, weak magnetic fields applied across the temporal lobes to produce fundamental experiences such as the "sensed presence" (the feeling of a "Sentient Being") as well as their capacity to reduce depression and pain.

Neuroscience is predicated on the assumption that all experiences are so strongly correlated with brain activity and structure that they can be considered causes of both public and private behaviours. These are behaviors that can be observed or measured by others or observed ("measured") only by the experients. The latter are traditionally labeled as thoughts and ideas. The recent documentation of the Default Mode Network [2] [3] that includes the anterior cingulate, posterior cingulate, posterior temporal-parietal lateral region and related structures as well as other highly predictable networks for specific types of cognitive operations, has indicated that the traditionally obscure domain of "thinking, beliefs, and exotic experiences" is caused by brain activity. Consequently they are measureable. If they are measureable then the responses to experimental manipulation can be discerned, categorized and simulated.

If all experiences are caused by specific complexities of brain activity then affecting this activity must potentially modify any specific class of behaviors. The consequence of brain injuries, whereby local damage or loss of neurons disinhibits neuronal clusters in other regions which actually cause the emergent behavior, is well known. The discernment of subtle more complex changes requires more precise instrumentation. Since the implementation of imaging technology such as fMRI (functional Magnetic Resonance Imaging), SPECT (Single Positron Emission Computerized Tomography), PET (Positron Emission Tomography), quantitative electroencephalographic (QEEG) and s-LORETA (standardized Low Resolution Electromagnetic Tomography) the data have clearly shown that routine operations such as presenting different stimulus patterns through different sensory modalities produce predictable and reliable alterations in activations of networks within the human cerebral volume as well as very predictable private and public behaviors.

As indicated by Johannes Mueller during the late $19^{\text {th }}$ century, human awareness was effectively a reflection of "the states of the nerves" rather than the direct isomorphic representation to stimuli impinging upon the senses. Elementary neuroanatomy indicates that there are at least six transformations, represented as synapses that occur between the impingement of photons upon rods and cones and the experience of a gestalt image at the level of the cerebral cortical networks. As aptly shown by Van Essen and Drury [4], the information contained within the action potentials from what appears to be a singular retinal image is deconstructed upon arrival within the caudal cerebral cortices into multiple components that include position, color, motion, shape, and meaning. These fractal components are then reintegrated into the perceptions that are experienced as a "whole" or gestalt.

As a result inexplicable phenomena such as palinopsia [5] are no longer mysterious but represent the integration of additional information into the aggregate before it is manifested as experience. The source of the extraneous patterns of neuronal activity that could become integrated into the final gestalt that constitutes the perception is usually ascribed to endogenous anomalous networks. They can also be the source of "illusions" or hallucinations. However the occurrence of this "re-integrative process" at the cerebral cortical level suggests that other stimuli such as electromagnetic fields applied externally from sources that penetrate the cerebral volume could also be integrated into the final experience.

The experimental and medical capacity to modify all brain activity and hence experiences has the potential to open new domains for treatment and explanation. One of the most powerful and persistent experiences (which 
are correlated with the activity within the caudal cerebral space) and beliefs (which are strongly correlated with the prefrontal regions associated with the organization of experiences) involves phenomena that are described as "sentient". They are experienced as occupying a larger space and longer time than the individual experient. This Sentience is considered personal and strongly emotional [6]. The Sentience or its anthropomorphic attributions (the Sentient Being) is perceived as intricately related to the person's sense of self as well as the persistence of this sense of self after the demise of the brain and body.

In clinical settings the concern about death and the disruptive consternation associated with the demise of the person is often a major pre-occupation. Some philosophers and early $20^{\text {th }}$ century clinicians [7] had suggested that thanatophobia, that is the fear of death, was the source of all anxieties. Anxiety is defined operationally as the anticipation of an aversive stimulus such as the unknown following dissolution of the self. Unbridled anxiety results in difficulties with adaptation and can significantly reduce the patient's capacity to achieve the universal goals of clinical normality. They are the ability to live relatively free of distress, the capacity to love and to be productive, and the ability to develop the person's own unique potential.

The concepts of Sentient Beings (the anthropomorphic attribution of the sensed presence) whose temporal duration is indefinite and who occupy all of space imply cognitively that there is no finality. If a person can be perceived to be a subset or identity with this "non-finality" then anxiety does not occur because there is no end. If belief concerning a Sentient Being is acquired by cultural learning or following a personal experience then the personal anxiety can be reduced. Like other forms of negative reinforcement, any response that is associated with the removal of a negative stimulus will be repeated. The cognitive operation for reducing this potentially devastating existential anxiety is remarkably simple. It is usually reflected in some statement such as "I am child of X" or "I believe in X" where X is the cultural name for the Sentient Being. If personal anxiety is reduced, the probability of displaying these verbal behaviors both as thoughts and speech are more likely to occur again [8]. Subsequent reduction of depression and re-integration of the person's cognitive structure with purpose and productivity, without medication, following these conversions have been remarkably ubiquitous both historically and cross-culturally.

The clinical and experimental capacities to directly affect the cerebral processes that are central to the experience of a Sentient Being or a Sensed Presence creates the potential to implement this technology for amelioration of distress experienced by patients who are terminal, who are engaging in protracted and unresolved grieving, or who are experiencing incapacitating existential anxiety. For the last 30 years we [9]-[11] have been developing a technology to accommodate these persistent human conditions that could be employed by the clinician to improve the quality of life. The technology is based upon the basic principles of Neuroscience. If all experiences are produced by specific brain activity and structure then the simulation of these patterns of activity within the cerebral volume should produce these experiences.

The emergence of a domain of research which has been most frequently labeled as "neurotheology" [12] is a logical extrapolation of the premise that all experiences are produced by brain function. However because the subject matter, that is the beliefs about the validity and sources of the subject matter (theological entities) are subject to testing and potential refutation, one would expect frustrative aggression to be displayed by strong believers when the validity of their beliefs is challenged. In this context the scalar quantity is more critical than the vector. In other words it is not the type of belief, theism or atheism; it is the magnitude of that belief that would be expected to produce the greatest frustrative aggression and opposition towards the idea. Although the arguments of antithesis are consistent with Hegel's interpretation, arguments based upon affect and agenda rather than facts and data do not often contribute to the productive synthesis that allows beneficial application to Science and Medicine.

\section{The Discovery}

Like many discoveries the elicitation of the sensed presence by physiologically-patterned, weak magnetic fields was based upon an intuitive challenge. The left hemispheric processes were associated with the sense of self [13], mediated primarily through linguistic operations. The sagacious insights of Durkheim [14] reflected in his $19^{\text {th }}$ century book, Suicide, indicate how the sense of self and its integrity is influenced by language and culture. From this perspective the proclivity for human beings within a culture to fight or to die to maintain their language and culture is not only predictable but very likely strongly determined. This approach also indicates that the sense of self is intricately interwoven not only with the process of language operations within brain functions but also with the idiosyncratic details of the language's syntax and semantics. 
The obvious question is: what analogous experience is associated with right hemispheric processes? The prescient and perspicacious $19^{\text {th }}$ century neurologist Hughlings Jackson [15] had inferred, on the bases of observing many patients before and during temporal lobe seizures, the transience of a "parasitic" consciousness that was experienced as "the other". Many other researchers such as Julian Jaynes [16] as well as Suedfeld and Mocellin [17] have emphasized the importance of "the sensed presence" that can be produced by specific environmental factors or altered states. When we applied physiologically-patterned fields across the temporal lobes such that there was a slight gradient from the right (higher) to the left side a sensed presence was frequently reported [18]. However like the natural or spontaneous occurrences of the sensed presence there were concurrent environmental conditions required to enhance the probability that the experience would be reported. After all, the sensed presence or its attribution to a Sentient Being is not a frequent or routine experience for most human beings.

There were several conditions required to produce the effect. First, physiologically-patterned magnetic fields were essential. By "physiologically-patterned" we mean those with temporal shapes and characteristics that imitate or simulate the electromagnetic changes of single neurons or aggregates of neurons within the cerebrum. Second, the person must sit blindfolded within a dark acoustic chamber or room. We reasoned that without the passive stimulation from background sounds and sights the neurons that would typically be involved with responding to these stimuli would then have the capacity to be recruited into the patterns induced by the experimental fields penetrating through the cerebrum. Consequently, the intensities of the applied fields were not required to be excessive. We assumed that the energy associated with the patterned magnetic field within the cerebral volume was the primary basis of the mechanism rather than frank current induction most typically defined by Faraday's law. Within the cerebral space the effective intensity ranges were found to be between 100 nanoTesla and 5 microTesla. This magnitude is a million times less intense than that employed for TMS or transcranial magnetic stimulation [19] that operates through current induction within focal areas. The two mechanisms are quite distinct but can produce similar effects. To differentiate the two methods we have sometimes referred to our technology as "Transcerebral Magnetic Stimulation (TCMS)".

The third component was the manner in which the magnetic fields were generated. Function generators which produce temporally symmetrically sine waves and square waves were not effective or minimally effective. Instead the computer software-generated magnetic fields developed by Professor Stanley A. Koren required point durations that when integrated over time produced the physiologically-patterned fields [20]. Point durations of 3 ms were more effective than either $<2 \mathrm{~ms}$ or $>4 \mathrm{~ms}$. This meant that great care was required to ensure the pattern was not distorted. This is analogous to ensuring the structure of a molecule in neuropharmacology. If a component, even a single chlorine or amine group, is altered or deleted many molecules no longer function within the brain in the same manner. Fourth, the total duration for exposure was about $30 \mathrm{~min}$. Briefer exposure durations were less effective. Fifth, it was optimal, except for film crews who were documenting the phenomenon, for volunteers to be "blind", that is not aware, of whether or not they were receiving actual fields or no fields (sham condition).

The standard protocol is to explain to the person that relaxation during the exposure without cognitive direction is optimal. Talking in order to communicate the experiences (through a lapel microphone to the experimenters) might interfere with the phenomenon but the person is free to do so if he or she wishes. Each subject is told that the experiences are subtle and that he or she should behave as a passive observer rather than actively intending. Two patterns of fields produce the most frequent numbers of sensed presences. The first is a $30 \mathrm{~min}$ exposure to a frequency modulated (decelerating) pattern with $3 \mathrm{~ms}$ point durations such that the range shifts between $26 \mathrm{~Hz}$ and $8 \mathrm{~Hz}$ once every $2 \mathrm{~s}$ continuously. This has been also called "the Thomas pulse".

At the end of the 30 min sequence the person responds to a questionnaire containing 20 items that refer to the typical experiences a person might perceive during the exposure. The details of the items have been published elsewhere [21]. The questionnaire is completed in dim light while the person is still wearing the Koren Helmet ("the God Helmet") that generates the fields across the temporal lobes and is sitting in the comfortable chair. The person is then blindfolded again. For another $30 \mathrm{~min}$ a second pattern (accelerating frequency modulated pattern) is presented with equal intensity across both hemispheres for $\sim 1 \mathrm{~s}$ every $3 \mathrm{~s}$ or $4 \mathrm{~s}$ while the person sits in the darkness in the acoustic chamber. At the end of the $30 \mathrm{~min}$ the same questions are answered again. The particular mark-space ratio of $1 \mathrm{~s}$ every $4 \mathrm{~s}$ was derived from the results of experiments involving weak electrical currents that showed a stimulation once every $4 \mathrm{~s}$ resulted in analgesia. The presentation of two patterns allowed each person to serve as his or her own control with respect to the differential effects of the fields. Pre- 
vious experiments [22] have shown that the reversal of the order of presentation of the two types of fields is associated with minimal reports of a sensed presence. We consider the critical nature of the order similar to many pharmacological phenomena. For example if lithium chloride is injected into rats 4 hrs before injections of 30 $\mathrm{mg}$ per $\mathrm{kg}$ of pilocarpine, clear limbic seizures with overt convulsions emerge within about $30 \mathrm{~min}$. If the injection order of the two compounds is reversed, neither seizures nor convulsions occur [23].

Based upon the results of the questionnaire the experiences associated with the first $30 \mathrm{~min}$, when a continuous $3 \mathrm{~ms}$ point duration, frequency modulated (decelerating) pattern with slightly higher intensity is applied over the right hemisphere are more negative and tend to be lateralized or at least attributed to the left side of the body. If fear is to be reported it occurs during this interval. During the second $30 \mathrm{~min}$, the theme of the report is usually more relaxing tranquil and peaceful. Frequently (although not always) the sensed presence is more likely to occur or is more intense during this segment. What we have learned over the last 30 years is that people who have experienced negative or untoward effects from consuming morphine also reported that exposures to these magnetic fields were unpleasant. Even in the popular literature reports of mystical experiences are not always positive. In fact about $10 \%$ of near-death experiences, the prototype for positive sensed presence phenomena, are extremely aversive [24].

In recent years the person wears an EEG cap under the helmet which allows continuous real-time monitoring of the person's QEEG for later analyses by s-LORETA. The helmet is a modified snowmobile head piece within which four pairs of solenoids (275-0232 Radio Shack reed relays, 5 VDC, 250 ohms; nominal current $20 \mathrm{~mA}$ ) are embedded in the sides at the level of the temporal lobes. The circuit is connected such that at any given time a solenoid on the left and right side of the helmet is activated; consequently the magnetic field is generated through the space occupied by the participant's cerebrum. The particular field pattern is rotated to each of the four pairs of solenoids every $0.5 \mathrm{~s}$ such that a complete cycle is completed every $2 \mathrm{~s}$. Because of the orientation of the applied field, the low strengths, and the nature of EEG filtering there are no induction artifacts. They can be produced by increasing the applied field strength. When they are present, they are obvious.

The different types of experiences associated with the sensed presence have been described elsewhere [25]-[27]. Factor analyses have revealed a gender structural difference to the items that are correlated with the sensed presence. Although there are always individual signatures, the general pattern of the themes associated with our protocol are consistent when they occur. Reference to a "presence" is reported about $80 \%$ of the time. The patterns of experiences are also frequently induced when volunteers are exposed to similar patterns but generated by different equipment that was designed by the second author. They have been called the Shiva and Shakti series.

In the clinical setting the efficacy of a component of the protocol has been demonstrated and replicated. Baker-Price and her colleagues [28] [29] exposed patients who had sustained closed head injuries and were experiencing pain and depression that were refractory to anti-depressant medication. Patients were exposed to the burst-firing pattern (the second pattern in the standard protocol) for one hour once per week for 6 weeks. In both series of clinical trials the patients who were exposed to the burst-firing transcerebral magnetic fields reported significant reduction in depression that was verified by psychometric data. There was also reduction of pain that was so conspicuous that some patients requested their own devices for their personal use. Patients exposed to sham field conditions either did not report this improvement or did not complete the trials. Dropouts from clinical trials where voluntary participation is the only motivation are often indicative of failure for the desired effect to occur.

That the fields were indeed affecting nociceptive thresholds and not produced as an artifact of the procedure or placebo was indicated by comparable reductions in pain thresholds displayed by rodents [30]. Rats exposed for $30 \mathrm{~min}$ after a baseline nociceptive threshold measurement to the burst-firing magnetic fields displayed marked elevations of threshold (analgesia) that was comparable to a single injection of $4 \mathrm{mg} / \mathrm{kg}$ of morphine sulfate. The analgesia from the magnetic field exposure was still apparent $30 \mathrm{~min}$ after the termination of the field exposure when the third threshold measure was taken [31]. The effect was evident over three successive days of treatments. The physiologically-patterned induced magnetic field analgesia could also be blocked by the mu-receptor antagonist naloxone which indicated that the field was mediating its effect through the same or similar receptor-coupled chemical pathways as morphine [32].

Martin and his colleagues [33] showed that rats that had sustained brain injuries, secondary to experimentally induced limbic seizures, responded to the positive effects of $30 \mathrm{~min}$ exposures per day for 3 days. The braininjured rats also showed a permanent shift in their nociceptive thresholds. Over three successive days the base- 
line threshold for nociception for these rats returned to that of normal rats that had never sustained brain injuries. This type of long-term change would be consistent with the persistence of the diminished pain and depression that was reported by our patients after they were exposed to our standard treatment. Their symptoms had not been responsive to tricyclic and SSRI antidepressants. Many volunteers who have experienced a powerful sensed presence during exposure to our magnetic field protocols later reported there were persistent changes in their beliefs and world views that were still manifest years after the single 60 min exposure.

\section{Responding to the Antithesis Arguments: The Terminology}

There have been a significant number of online misunderstandings and misrepresentations of our research and approaches concerning "neurotheology". Our major goal has been to develop technologies that will aid in the patient's or client's personal development within the domains that have often been reserved for Sentient Beings and the ultimate meaning for the sense of self and its dissolution or demise. Aaen-Stockdale's magazine article [34] is one example of the proliferation of multiple errors. According to Aaen-Stockdale "As far as I can tell, Persinger's theory is based on the literature on religiosity in temporal lobe epileptics; a literature that I argue ... is both flawed and outdated".

He refers to an experiment by Booth and Persinger [35] that reported the measurements of discrete shifts within the theta band of QEEG between the frontal and parietal regions of the right hemisphere and the experience of the sensed presence. This experiment did not involve temporal lobe epileptics nor did the effect require the reference to or the presence of complex partial epilepsy. The results of the experiment, that have been replicated many times, involved the sensed presence, which is an experience. It is not religiosity, which relates primarily to shifts in behavioral patterns ("personality”) and beliefs. The distinctions between experiences and beliefs are important because they involve different networks of cerebral activity and imply different strategies for intervention.

The literature concerning temporal lobe epileptics and religiosity to which Aaen-Stockdale referred involved the classic studies by Dewhurst and Beard [36], Slater and Beard [37] and Bear [38]. There have been multiple case studies where the sensed presence or the feeling of someone standing nearby was associated with a right temporal lobe anomaly such as a space-occupying lesion. In our clinical practice these cases are occasionally evident during routine EEG following neuropsychological assessments. Persinger and Tiller [39] described a woman who reported a frequent right-sided presence preceded by the sensation of "an electric shock" through either her right hand or both hands, icy coldness, and vibrations moving through her body.

The presence was considered profound and was associated with paroxysmal $4-5 \mathrm{~Hz}$ activity over the temporal lobes. In the original case [40] that initiated the first author's interest in the phenomenon almost 40 years ago a seasoned transcendental meditation instructor reported a profound presence of god that suddenly appeared beside her in the laboratory towards the end of a routine $20 \mathrm{~min}$ meditation. The very meaningful experience was concurrent with conspicuous spike and slow wave activity for about 10 to $20 \mathrm{~s}$ over the right (T4) temporal region. The fundamental themes from these types of clinical observations have been replicated by direct electrical stimulation of deep (mesiobasal) and cortical temporal lobe structures by Kubie [41], Williams [42], Halgren [43], Stevens et al. [44], Bancaud et al. [45], Gloor [46] and Mahl et al. [47].

These were classic studies whose relevance and validity are still clear today. In fact Picard [48] identified an activation source within the right dorsal mid-insula (where it converges with the temporal stem) during intense feelings of meaningfulness and bliss. The association is not specific to patients. Persinger and Makarec [49] completed factor analyses of responses to a questionnaire whose construct validity for temporal lobe functions [50] had been verified. They found that the feeling of a "spontaneous presence" loaded on the same factor as a history of transient episodes of enhanced verbal meaningfulness associated with "cosmic" attribution. Even with strip chart EEG profiles the correlation between increases in relative right temporal lobe theta activity, Vingiano's hemispheric quotient, and a history of reported sensed presences [51] was apparent. As the technologies for direct measurement of behavior and brain function have become more precise, the intricate relationships between the sensed presence and its various manifestations in a person's personality and experiences have been verified. Urgesi et al. [52] reported that modifications of neural activity in the temporoparietal area could induce changes within the "spiritual brain" that leads to self-transcendence. Within the brains of individuals diagnosed as "schizophrenics", according to Taber et al. [53], the process could promote misattributions and religious delusions.

The more recent MRI imaging studies have consistently confirmed the clinical observations by Dewhurst and 
Beard [36] that sudden religious conversions are related to alterations in the right hemispheric cerebral circuitry often found in complex partial epileptic seizures. Wuerfel et al. [54] measured an inverse relationship between religiosity and right hippocampal volumes in patients with refractory epilepsy. Significantly greater hippocampal atrophy was also reported by Owen et al. [55] in older, non-epileptic adults who had reported life-changing religious experiences. In fact Chan et al. [56] have suggested that hyperreligiosity, visual hallucinations, and cross-modal experiences may be a syndrome relatively specific to right temporal lobe atrophy. Because the process contributing to the atrophy or reduced hippocampal volume would facilitate the electrical lability of the right temporal lobe intermittent intercalation between the two hemispheres and experience of the right hemispheric equivalent (the sense of presence or Sentient Being) of the left hemispheric sense of self would occur more frequently. As predicted by our hypothesis such cerebral organizations are the central bases for these experiences spontaneously and can be simulated or evoked within the laboratory when the appropriate intracerebral electromagnetic patterns are simulated.

The primary limit of Aaen-Stockdale's argument is he states that we have assumed that religious experiences are epileptic events. This is not correct. We have reiterated that many of the experiences reported by individuals who report intense religious experiences involving a Sensed Presence or a Sentient Being are similar to the experiences reported by individuals who have been diagnosed with complex partial epilepsy with a focus within the temporal lobe. The experiences during the specific, limited interval of the ictal display must be differentiated from the interictal behaviors that often emerge subsequent to the multiple ictal events. Like the prototype of "kindling" the gradual changes in synaptic re-organization within limbic structures (particularly the dentate gyrus) results in permanent alterations in behaviors. The latter are more associated with what Bear [38] labeled as the "temporal lobe personality". These characteristics include hyper-religiosity, a sense of the personal (of being "selected" for a purpose), widening of affect, cognitive viscosity, circumlocution of cognitive processes, and a compulsion to proselytize about the universal significance of the (religious) experience. We have found this pattern of behavior can occur following energetic conversion to either theism or atheism. Both are often associated with a tireless and often aggressive compulsion to proselytize for "the good of Humankind".

In the first author's original hypothesis [57] from 1983 the term "microseizure" was employed as a metaphor to indicate the cerebral bases for the sensed presence and that it could interact with the substrates that determine the strength of endorsement of god experiences and beliefs in different cultures. The attributes of the deities reflect the expectations of the cultural beliefs and the reinforcement histories of the individuals. That the degrees or proportions of belief in a god, the average importance of that god and religiosity are related to cerebral function has been shown by Kanazawa [58] who correlated indices of these propensities with the general level of intellectual functioning within approximately 192 nations and regions. There were strong inverse correlations between the three belief variables and intelligence. He concluded that "national IQ alone accounts for more than $70 \%$ of the variance in the mean importance of God across nations".

That does not prove that belief in religious content involves diminished intellectual level. However it does indicate that what has been considered ephemeral or beyond scientific explanation, such religiosity, can be related to a measure such as intelligence that has been shown to have strong cerebral determinants. As reviewed by Fingelkurts et al. [59] the discipline of Neuroscience can now ask if the human brain is "hardwired to produce God" or is the brain "hardwired to perceive God". There is no requirement for pejorative inferences or conclusions. More recent direct measurements by Kanai et al. [60] have shown that even cognitive styles between liberals and conservatives with respect to political attitudes are discernable by differing MRI-measurable volumes of grey matter. They found that greater liberalism was associated with increased proportions of grey matter in the anterior cingulate while conservatism was associated with increased volume of the right amygdala.

The initial concept [57] of the "microseizure" was based upon normal behavior. Perhaps a more accurate term would have been paroxysmal discharge or pattern. For example, the depth recording of the limbic system of human beings during dreaming or rapid eye movement shows transient patterns that would be considered microseizures if they occurred over the cerebral cortices during the day. Having assessed more than 1000 patients who sustained a closed head injury from concussive impacts, without loss of consciousness, over the last 35 years the first author has noted that the occurrence of transient paroxysmal activity for about 10 to $20 \mathrm{~s}$ over the right temporal lobe is almost always associated with a concomitant experience of a sensed presence. The person is not "epileptic". The experience and the unique EEG signature may never occur again.

Clinical neurology indicates that even when patients are diagnosed as complex partial epileptics with a focus in the temporal lobes overt anomalous displays can occur without discernable electroencephalographic patterns. 
This would be consistent with the seminal observation of Goldensohn [61] who implanted electrodes within the cerebrum of epileptic patients and compared the activity with those from the classical international 10 - 20 sensor array. The electrodes embedded within the cerebral cortices were separated by about $2 \mathrm{~mm} ; 4$ to $6 \mathrm{~cm}$ usually separates surface sensors. He found that paroxysmal activity was frequently measured from the embedded electrodes while no activity was evident from surface EEG measurements. In addition he found that shared paroxysmal activity within a critical density of the $2 \mathrm{~mm}$ electrode arrays was required before a representation was detected by the surface indicators. Goldensohn attributed the "microseizures" to labile activity of cerebral cortical columns which in the human brain are about 0.5 to 1 millimeter in diameter. When a critical number of these cortical columns were activated the clinical indicators were then discernable.

The concept of a continuum has been central to our approach. We have employed the term "temporal lobe continuum" to refer to the range of labilities (lower thresholds or greater "sensitivities") in the activities of the temporal lobes. Based upon questionnaire studies, the normal population is distributed along this continuum. The idea is similar to that developed by Richards and his colleagues [62] for individuals who have sustained closed head injuries from primarily concussive events. They have applied the term "epileptic spectrum disorder". This continuum concept is representative of the human population. The frequency (incidence) and intensity of spontaneous sensed presences as well as the cultural-specific anthropomorphism (Sentient Beings) also exhibit what appears to be a Gaussian-like distribution. Some sub-populations may report the experience of the propinquity of spiritual entities several times per day [63].

Direct measurements within human limbic structures have shown a clear neurodynamic bases for this continuum. Babb et al. [64] implanted (stereoencephalography) arrays of electrodes within the cerebra of epileptic patients and quantitatively assessed the proportion of coherent activity between the electrodes. During background conditions about 7\% of the electrode activity was coherent. During the "altered states" or what Hughlings Jackson would have likely called "dreamy” states when the "parasitic" consciousness of "another" would have been discerned, the coherence was 14\%. When the coherence achieved levels of about 36\% overt symptoms, such as clinical seizures and loss of consciousness, were reflected in the surface electroencephalographic activity. The cell signaling network underlying epileptic behavior, as reviewed by Bozzi et al. [65], involves many of the same pathways that are influenced by the specific patterns of magnetic fields by which we induce the sensed presence and produce long-term improvements in depression and reduction in pain.

\section{Responding to the Antithesis Arguments: Specific Applications}

Anecdotal accounts are precarious arguments for either support or refutation of a concept or a hypothesis. A more reliable source of support or refutation is based upon large groups of individuals such that the major source of variance for human behavior (individual differences) can be accommodated. Aaen-Stockdale states “Atheist-in-Chief Richard Dawkins was subjected (sic) to the God Helmet as part of a 2005 BBC Horizon documentary ("God on the Brain”). Afterwards Dawkins said: "It pretty much felt as if I was in total darkness, with a helmet on my head and pleasantly relaxed”. Not exactly a road to Damascus experience, but Dawkins is of course, a damned skeptic (sic) and Persinger simply argued that he wasn't temporal-lobey enough”. Although the statement may have been intended to portray a pique post-hoc rationalization, ignoring the temporal lobe dynamics of a person who is being exposed to the physiologically-patterned magnetic fields would be analogous to stating that a drug has no valid effects or is simply a placebo because a subset of patients do not express the receptor subtypes required to sequester the drug in order to mediate its effects.

We were the researchers who measured Dr. Dawkins directly (rather than being dependent upon second hand accounts). Dawkins did report significant experiences that are a component of the continuum that leads to a sensed presence within the experimental setting. He reported a mild dizziness, twitching in his legs, and a twitchy feeling in his breathing. These are the first types of experiences that are the antecedents to a profound sensed presence. Dawkins reported these experiences despite the fact that was required to sit for more than an hour while being exposed to hot lights and sounds while the BBC director adjusted conditions for filming. The first author wrote in his notes during the filming that the subject (Dr. Dawkins) was becoming increasing agitated from the protracted delays and discussions. In addition the original plan for the experiment was to directly compare Dr. Dawkin's experiences with those of the Archbishop of Canterbury who decided not to participate.

We know antecedent noisy and sensory overloading conditions before the exposure to the protocol is counterproductive to producing the state that allows the interaction with the applied transcerebral magnetic fields and 
is associated with the report of a sensed presence. Our actual protocol involves the subject sitting within the darkened chamber shortly after completing the consent forms and beginning the experiment within about 10 min once the scalp sensors and helmet are applied. Although now we employ the QEEG cap system so that the data can be transformed into s-LORETA images to discern where in the cerebrum the frequency-specific activities were changing during the experience of the sensed presence, the initial 3-channel (Grass Model 79) monitoring was to discern the arousal level of the subject. We had learned that if the person enters light sleep (Stage II) where prefrontal spindles emerge or if the person is too activated (dominant beta activity with little posterior alpha rhythms) the optimal interaction with the applied transcerebral fields does not occur as indicated by the subjective experiences and even the discernable alterations (by the older strip chart EEG technologies) over the right temporal regions.

For every negative anecdotal experience, there is a very positive anecdotal experience. This is consistent with Hegelian process. The televised case of one of our experiments involving an undergraduate female university volunteer for an episode of Morgan Freeman's Through the Worm Hole is an example. The subject reported the classic richness of the experiences associated with the sensed presence, out of body experience and the lateralization of the visual experiences to the side of the body that was dependent upon the major source of asymmetry of the applied field. Within a week we received a telephone call from one of the pioneers of deep surgical stimulation, Dr. Vernon Mark, who indicated he was struck by the profound similarity of what his patients had reported during surgical stimulation and the report of the subject in the Worm Hole episode during the application of external, weak transcerebral weak magnetic fields.

Like the continuum of temporal lobe lability or Roberts' epileptic spectrum disorder [62] there is a range of responses to the $60 \mathrm{~min}$ of exposure to the transcerebral stimulation of our standard protocol. One classic example was the young diabetic woman who experienced a sensed presence. When she attempted to discern the location of it, the presence appeared to move to the other side. We have since shown that when people intend the network pattern of brain activity change and consequently the vectorial characteristics of the interaction with the applied field changes. The woman felt a profound sense of peace and tranquility. When we stopped the field application, without her knowledge, the sensed presence faded away and she began to cry. She reported she felt as if she had lost a sense of closeness with a Sentient Being.

For any discovery to be sufficiently generalized to have utility for clinical treatment or intervention, the results cannot be anecdotal. The exit questionnaire data indicated that approximately $80 \%$ of the more than 500 subjects who have been exposed over the last 30 years to the appropriately patterned magnetic fields report "yes" to the item that they experienced a sensed presence. Post-session interviews indicated how the person explained or attributed the source of the presence. They varied as a function of his or her culture and beliefs. We have been impressed by the profundity and "realness" of the induced experiences. Even though all of the subjects know they are sitting in a chamber involved with an experiment it is not unusual for the person to attribute the experience to a deceased relative, a spiritual icon, or to a mystical source. Of the approximately 40 atheists we have tested, most of them reported both a sensed presence and an out of body experience. Like Michael Shermer, the editor of Skeptic, who was exposed to our standard protocol they attribute the experiences to their brains rather than to external mystical sources or culturally religious deities.

\section{Responding to the Antithesis Arguments: The Issues of Replication, Confounding Variables and Construct Validity}

Replication and the capacity for the average clinician or scientist to reproduce a phenomenon or effect is the cornerstone of Medicine and Science. Replication often reflects both the generalizability of the phenomena between places as well as the ease of reproducing the equipment that produces the phenomena. In the history of Science and Medicine there have been instances where the technology was so complicated or intricate, such as Leeuwenhoek's lens for the first microscope, that decades passed before others developed comparable technical capacities. Sometimes the idiosyncratic characteristics of the technology that created the effect may change over time inadvertently because of altered manufacturing and the phenomenon no longer appears. This has occurred many times in the chemical industry. For example after the manufacturing and synthesis techniques for coal tar derivatives such as dicyanin-A changed between the first and second World Wars, the diagnostic potentials for solutions of these compounds were no longer evident.

According to Aaen-Stockdale, "unfortunately the only published attempt failed to evoke a 'sensed presence'. 
Using a kit and code borrowed from Persinger himself Granqvist and his colleagues could not reproduce the effects”. What is not stated objectively is that Granqvist and colleagues [66] did not reproduce the effects because they did not reproduce the procedures. As reiterated by Persinger and Koren [67] the Granqvist group borrowed a prototype of another device that was not the helmet. They had told us they required a device they could use to discern if weak magnetic fields could affect profiles from Positron Emission Tomography. In addition they employed a computer and software that distorted the patterned field. The failure to elicit the effect would be analogous to changing the molecular structure of a ligand or a specific drug and then being surprised that the modification did not produce the same effect as the original ligand or drug. When the actual scores for the exit questionnaire reported by Granqvist's experimental and control groups were compared to the groups exposed to our sham field treatments, there was no statistically significant differences. In other words different populations tested by different experimenters with almost antithetic approaches to the phenomena displayed comparable experience scores following sham-field conditions. In most clinical trials this would be considered strong evidence that the treatment was not appropriately applied or elicited.

Although innovation and modification of a technique for the local requirements of the clinician or scientist support the generalized nature of a treatment, there is still a tacit assumption that the original protocol and construction of equipment will be followed. If replications don't occur when other equipment is applied the information becomes very useful for isolating the effective components of the equipment and procedure that produced the effect. For example French et al. [68] applied the "Goldwave" auditor editor to produce a wave-file version of one of our electromagnetic patterns. He asked the subjects to discern if they could detect the field or could sense a presence. They did not. Normally this would suggest that the appropriate equipment was not applied rather than dismissing the validity of the phenomenon. The second author had also employed the Goldwave procedure during the first draft of signal production. This method was not effective. The Murphy signals for the Shakti and Shiva equipment only became effective after a proprietary method was added. The validation of their efficacy and comparable impact with the Complex Software applied through the Koren Helmet was then demonstrated by quantitative electroencephalography by Tsang et al. [69].

Actual replications employ the equipment and measurements that are consistent with a phenomenon. If a clinical trial was intended to discern the efficacy of a new anti-depressant drug, measuring the patient's height and weight may not be revealing. Yet when Gendle et al. [70] tested the second author's 8-coil Shakti system to test subjective emotional experiences they used psychometric measures that were both non-specific and peripheral to the spontaneous experiences reported by hundreds of participants that were recorded in on-line monitoring protocols for years. These types of responses by other researchers are consistent with and predicted by Hegel's approach to discovery.

Double-blind studies for human subjects are considered important when cognitive structure is a significant variable in the outcome of an effect. Double blind studies are less required for drugs like strychnine where the expectations of the person have minimal effect on the outcome. In all of our major experiments involving the experimental induction of the sensed presence [e.g., 71], the procedures were double blind despite the insistent claims by Granqvist and their proliferation by Aaen-Stockdale [34]. By double blind we mean that the person was: 1) recruited to be in a "relaxation study"; 2) told they may or may not be exposed to a magnetic field (a requirement of the guidelines for the ethical treatment of subjects since the Nuremberg and Helsinki agreements), and; 3) exposed to different patterns of fields that elicited different degrees of producing sensed presences.

The experimenters, who were usually fourth year thesis students or graduate students, may have been aware of which subjects received the field or non-field once the volunteers were secured in the acoustic chamber, but they (the experimenters) did not know what type of field was being applied or whether it was a presence-inducing field or a "comparator field". That was known by the first author or another graduate student who was never present during the experiments. This is a standard procedure for controlling for placebo effects during drug trials. Even though we have repeated these facts multiple times, Granqvist et al. [71] and Aaen-Stockdale [34], as predicted by Hegel's concepts, do not incorporate the corrections into their discourse.

Although one could argue that our laboratory was known for studying the sensed presence, the fact that the subject did not know if he or she was receiving a field or what type of field was being received minimizes this confounding variable. Most of the subjects had completed a temporal lobe sensitivity and belief inventory (the Personal Philosophy Inventory or PPI) between one month and 10 months before the experiment. The long latency since the administration of the questionnaire would not have been sufficient to influence the immediate experiences during the experiment. Administration of the questionnaire was a component of the agreement with 
ethics board that if subjects said yes to question 113 "I have had an epileptic seizure" they would not be exposed to any of the magnetic fields.

Granqvist et al. [66] and French et al. [68] claimed they did not replicate the effects. Yet they did not manipulate the variables or attempt further replications through modifications of the parameters of their equipment. This is an established practice by in most laboratories because of idiosyncratic local factors and site-specific organizations of equipment that affect outcome. Instead both groups of researchers attributed the elicitation of the sensed presence by application of transcerebral magnetic fields to suggestibility. However neither group of researchers actually measured suggestibility. This characteristic was the first co-measurement completed in our original sensed presence studies. As reviewed by St-Pierre and her colleagues [71] we directly measured suggestibility for each individual subject by a clinical interactive scale developed by Spiegel and Spiegel [72] rather than infer this construct by secondary inferences from questionnaires as did Granqvist [66] and French [68]. Quantitative analyses indicated that when suggestibility was first covaried before the primary analyses of variance the effectiveness of specific magnetic field exposures to be associated with the report of sensed presences did not change significantly. Post hoc rationalization by enthusiastic explanations does not replace empirical and direct measurement.

It is important to emphasize the difference between primary effects and the potential role of placebo effects adding to or subtracting from a phenomenon. There is no doubt that the weak magnetic fields that elicit the sensed presence and reduce depression and relieve pain in human volunteers and patients also produce significant behavioral effects in non-human subjects which are presumably not prone to placebo responding. In addition to the powerful, duration dependent, "dose-dependent", and pattern-specific analgesic effects demonstrated for rats [31], planarian [73], and inferred from the behaviours of human subjects [74] while being exposed to these magnetic fields in our laboratories, comparable changes have been reported and replicated by many other research groups. Similar changes as well as receptor and pharmacological mechanisms have been shown for decades by Alex Thomas [75] and his colleagues. A thorough review of the dozens of experiments involving these fields was published by del Seppia and her colleagues [76]. During the year 2016 a commercial headphone device will be released (Nervana) that generates magnetic fields and sounds to produce analgesia. The manufacturer's claims are the effects are so potent that the user must accommodate at least 30 min of post exposure rest to ensure the effects have dissipated.

There have been replications of our original Koren Helmet effects by other researchers. For example, Tinoca et al. [77], a Brazilian research team, demonstrated a partial replication of differential emotive dimensions of verbal reports [78] when their custom-constructed device was applied over the temporal lobes. More than 20 years ago the remarkable innovative researcher Dr. Chris Montoya, a prior stem cell researcher from the University of Cambridge UK, utilized a custom-constructed helmet that synchronized pulsed light with similarly patterned magnetic fields. Powerful effects, that did not require questionnaires to be discerned, were induced within a population of 100 young adults from Williams Lake British Columbia. However, 10 years after the experiments were completed his current university's ethics committee prohibited him from publishing this research, coupled this censorship to his maintained employment, and argued that the paper's publication could impact other grants and scholarships from the Canadian Tri-Council. As predicted by Hegel, even the occurrence of results that are consistent with a thesis can be ignored or suppressed during the phases of antithesis. A further decade later he is now currently re-attempting to have this research published.

Construct validity has been considered by many scientists the quintessential indicator of the veridicality of a hypothesis. Construct validity suggests that one is measuring what is supposed to be measured. Our basic hypothesis is that the sensed presence is the right hemispheric equivalent to the left hemispheric sense of self. The two stages of the exposure protocol were designed to first activate the right hemisphere such that when the second field, which is applied more homogenously occurs the initial activation can more easily intercalate with left hemispheric processes. As a result the person becomes transiently aware of the right hemispheric equivalent of the sense of self. This is the sensed presence. The labeling and correlative imagery is a function of cultural expectations. The details and images of the "memories" of these experiences are determined by the active and passive (primarily left prefrontal) encoding at the time of the experiences. Most of the "details of the memory" a person reconstructs through right prefrontal activations days later are dominated by the images and details of the verbal labels that were applied to the experiences rather than the evoked experiences themselves.

Recently Saroka and Persinger [79] applied s-LORETA technology to discern the level of coherence between the left and right hemispheres during the protocol. The groups that reported a sensed presence differed from 
those who did not. The only statistically significant interhemispheric coherence occurred between the left and right temporal lobes as predicted by the hypothesis. There was increased coherence within the $10 \mathrm{Ho} 13 \mathrm{~Hz}$ band for those subjects who would ultimately report a sensed presence that began about 5 min after the initiation of the first field and continued for about $15 \mathrm{~min}$. About five min after the initiation of the alpha coherence between sensors T3 and T4 an increase in coherence for the gamma range (30 to $40 \mathrm{~Hz}$ ) occurred between the more caudal temporal regions at T5 and T6. s-LORETA confirmed that during the latter state there was a marked and conspicuous increase in current densities associated with gamma range power within the right prefrontal region and anterior portions of the temporal pole. These results were considered consistent with the concept that the sensed presence, when it occurs, is associated with enhanced intercalation between the left and right temporal lobes during which time the person becomes "aware" of the right hemispheric sense of self, i.e., the presence.

\section{Responding to the Antithesis Arguments: Importance of Mechanisms and Theory}

When mesmerism was the primary means of producing analgesia for surgery before the development of modern pharmacology, the consensus was there no viable mechanism. Therefore it could not be effective. During the $19^{\text {th }}$ century James Braid witnessed the powerful effect of this procedure during a popular demonstration. Shortly thereafter he demonstrated the same phenomena to his medical colleagues. He explained this "new discovery" according to the accepted mechanisms of the day and called it another name: "hypnotism”. By changing the name and offering a palatable mechanism that was consensual to the scientific peers of his day, a powerful phenomenon that had been denied since the French Academy of Science had dismissed it and its originator became legitimate.

The designation of a domain of heterogeneous phenomena with multiple and different mechanisms by a single word such as magnetism does not imply all phenomena within the domain are equal or the same. One of Edison's researchers placed his head for a few minutes within a strong magnetic field during the early part of the $20^{\text {th }}$ century and concluded there was no effect of magnetic fields upon the brain or thinking. However strong magnetic fields when appropriately applied produce significant alterations in organ states as well as their biomolecular substrates. Applied optimally they become the bases for MRI. When focused specifically the fields become the technology of Transcranial Magnetic Stimulation. Dismissing all potentially significant phenomena due to exposure to weak, physiologically-patterned magnetic fields by placing a static refrigerator magnet over the head because there was no subjective experience is analogous to asking the patient to drink dihydrous (mono) oxide and upon observing no effects concluding therefore all chemicals have no effect. The statement ignores the myriad nuances of temporal patterns that can resonate and interact with the matter that composes the brain and consequently the experiences generated by it.

The argument by Aaen-Stockdale that the weak magnetic fields cannot penetrate the skull (and hence the cerebral volume) was based upon belief rather than upon evidence. Complete penetrability of the same fields and point durations employed in the protocol through thicknesses (wood) more than twice that of the human skull was shown experimentally by Persinger and Saroka [80]. The field strengths were reduced by only about a third when sheet metal (for duct systems) was used as a simulated skull. The importance of measurement rather than speculation or "sensibility" cannot be over emphasized. Subtle energies can evoke powerful effects that are mediated from the basic units of matter to the complex organizations from which consciousness emerges. Chapellier and Matta [81] have also expressed this approach: "medium stimulation by adequate magnetic fields can modulate this phase shift in a therapeutic direction”.

The recent demonstration by the punctilious experiments by Buckner and her colleagues [82] clearly demonstrated that the sensed presence fields with specific point durations of 3 ms presented for 30 min altered the T-type calcium channels in plasma membranes of cells in culture. It has been known for more than a decade that only 30 min of intractable paroxysmal activity within the limbic structure in rats can produce permanent shifts in calcium channels within hippocampal neurons from L-type to T-type [83] which encourage burst-firing and interictal behaviors. Burst-firing patterns are fundamental to many critical phenomena within brain space including long term potentiation (the major substrate to the formations of “memories"), kindling, and the synaptic reorganization that produces the interictal environment [84] that supports the changes in personality described by Bear [38]. The coupling between T-type calcium channels to potassium channels in membranes of neurons that control dopamine release [85], the strong correlates to both addiction and subjective meaningfulness, suggests 
that the precise identification and synthesis of the molecular mechanisms for the ecstatic states of religious experiences and Dostoevsky-type rapture, and the physiologically-patterned magnetic field induction of these experiences [79] depend only upon the precision of the appropriate technology.

The other major argument is that only intense, Tesla-level magnetic fields can evoke changes in neuronal activity. From a faradic perspective, which is based upon mechanical induction of changes from the current produced from alterations in electric fields with respect to the resistivity of interstitial fluids, large field strengths are required to affect cell metabolism. The human cerebrum generates about 20 Joules per second (Watts) due the utilization of glucose to maintain the integrity of the cells and their processes. On the other hand the primary correlates of thinking and thought involve much less energy that has the potential to be influenced by much smaller quantities of energy [86] [87]. Metaphorically one can employ two methods to open a door. One can kick the door open with brute force. Or, one can open the door through a lock with a key. The latter requires understanding the pattern of the interaction and the match between the key and the lock. This is the same metaphor employed by the $19^{\text {th }}$ century physician Paul Ehrlich to describe the precision and small quantities of energy associated with the binding of the ligand to the receptor.

The action potential, the presumed basis of experiences that are determined by the patterns of those potentials, involves about $10^{-20} \mathrm{~J}$ [86]. The critical mass of neurons distributed through the cerebral cortices that are associated with "awareness" of experiences or a perception has been estimated to be in the order of $10^{7}$ [87]-[89]. Consequently if each of these neurons were generating a median 10 action potentials per second $(10 \mathrm{~Hz})$ the total energy would associated with "cognition" would be $\sim 10^{-12} \mathrm{~J}$. This quantity is more than a trillion times less energy than that required to maintain the integrity of the cellular metabolism. The energy from the temporally patterned magnetic field applied within the cerebral volume of about $10^{-3} \mathrm{~m}^{3}$ can be estimated by the product of the volume and the magnetic field strength $\left(1 \mu \mathrm{T}^{2}\right)$ divided by $2 \mu$ (magnetic permeability) where $\mu$ is $4 \pi \cdot 10^{-7}$ $\mathrm{N} \cdot \mathrm{A}^{-2}$. The result is $10^{-9}$ to $10^{-10} \mathrm{~J}$ which is a factor of 100 to 1000 more than required to accommodate the energy associated with the aggregate of action potentials.

The marked discrepancy between mechanically based and electromagnetic based energies is important to understand. If a $1 \mathrm{gm}$ candy was dropped from a distance of $1 \mathrm{~m}$ onto a table the force would be in the order of a milliNewton. The energy applied through the upper veneer of the table would be in the order of microJoules. As a force the effect is negligible. However if the energy were electromagnetic and exhibited a wavelength within the visible range, it would be sufficient to produce a sensation of light in the dark-adapted eyes $\left(\sim 10^{-17}\right.$ Joules) of every human being on this planet. The quality of the pattern is also critical. A person can experience the sensation of loudness by sound pressure fluctuations of only a few milliPascals. However there are no reliable accounts of people "hearing" the atmosphere whose quasi-static pressure is a billion times (101 kPa) more intense.

The fact that the human cerebrum can respond to different stimuli whose quantities of application can differ by orders of magnitude reiterates the versatility of the organ. Persinger and Saroka [90] compared the proportions of experiences and intracerebral consequences from the classical studies of surgical stimulation and exposures to the protocol that generates the sensed presence. Our analyses concurred with the impression of Vernon Mark of the remarkable similarity of the experiences despite the differences of method: current induction with imbedded electrodes vs. externally applied temporally patterned magnetic fields. There were specific differences that reflected the application geometry or the population. What was more critical was the calculated numbers of neurons that would have been activated by both methods. Employing the actual values of the electrical parameters for the surgical stimulation reported by the surgeons we found that numbers of neurons that would have been influenced were the same order of magnitude as the numbers of neurons that could be affected by the energy from our applied magnetic fields.

\section{Conclusion}

We have both written different texts [91] [92] regarding the neuroscientific bases for the powerful subjective behaviors that have been labeled "religious", spiritual, deities-related, or mystical experiences. These patterns of responses are predictable by the contemporary principles of brain function, particularly the properties within the right temporal lobe and the various intrinsic neuronal networks that strongly affect this region. The experimental simulation of the general class of experiences by applying weak, appropriately and physiologically-patterned magnetic fields across the temporal lobes has been replicated within and between laboratories. As predicted by Hegel's concept of challenges to contemporary knowledge the responses to the implications of this approach and 
technology have been characterized by derision, superficial arguments, or failure to apply the same methods of investigative persistence that are standard in other areas of endeavor. The final stage of the Hegelian process will be the synthesis of the opposing views that is likely to involve the integration of "neurotheology" with the "Rosetta stone" that ultimately allows the translation of electromagnetic energies to more well known particulate processes that define molecular pathways.

\section{Acknowledgements}

The authors thank Dr. Blake T. Dotta for his technical contributions. The publication of this research would not have been possible without the courage and dedication of Dr. Linda S. St-Pierre, the Laurentian University Faculty Association (LUFA) and the Canadian Association of University Teachers (CAUT) who have defended our academic freedoms and rights to publish the results of unpopular hypotheses and to express our opinions without censorship or retribution.

\section{References}

[1] Simpson, P. (1997) Hegel’s Transcendental Induction. SUNY Press, New York.

[2] Collins, M.W.G. and Persinger, M.A. (2014) Enhanced Power within the Default Mode Network in Normal Subjects with Elevated Scores on an Egocentric Scale. The Open Neuroimaging Journal, 4, 5-10. http://dx.doi.org/10.2174/1874440001408010005

[3] Luo, Y., Kong, F., Qi, S., You, X. and Huang, X. (2015) Resting-State Functional Connectivity of the Default Mode Network Associated with Happiness. Social Cognitive and Affective Neuroscience, 11, 516-524. http://dx.doi.org/10.1093/scan/nsv132

[4] Van Essen, D.C. and Drury, H.A. (1997) Structural and Functional Analyses of Human Cerebral Cortex Using a Surface-Based Atlas. Journal of Neuroscience, 17, 7079-7102.

[5] Mohr, J.P. and Pessin, M.S. (1992) Posterior Cerebral Artery Disease. In: Barnett, H.J.M., Stein, B.M., Mohr, J.P. and Yatsu, F.M., Eds., Stroke: Pathophysiology, Diagnosis and Management, 2nd Edition, Churchill Livingstone, New York, 419-441.

[6] Persinger, M.A. and Makarec, K. (1986) Temporal Lobe Signs and Correlative Behaviors Displayed by Normal Populations. Journal of General Psychology, 114, 179-195. http://dx.doi.org/10.1080/00221309.1987.9711068

[7] Hall, G.S. (1915) Thanatophobia and Immortality. The American Journal of Psychology, 26, 550-613. http://dx.doi.org/10.2307/1412814

[8] Persinger, M.A. (1985) Death Anxiety as a Semantic Conditioned Paradigm. Perceptual and Motor Skills, 61, $320-322$. http://dx.doi.org/10.2466/pms.1985.61.1.320

[9] Persinger, M.A. (1993) Vectorial Cerebral Hemisphericity as Differential Sources for the Sensed Presence, Mystical Experiences and Religious Conversions. Perceptual and Motor Skills, 76, 915-930. http://dx.doi.org/10.2466/pms.1993.76.3.915

[10] Persinger, M.A. (1994) Sensed of a Presence and Suicidal Ideation Following Brain Injury: Indications of Right Hemispheric Intrusions from Neuropsychological Profiles. Psychological Reports, 75, 1059-1070. http://dx.doi.org/10.2466/pr0.1994.75.3.1059

[11] Persinger, M.A. and Healey, F. (2002) Experimental Facilitation of the Sensed Presence: Possible Intercalation between the Hemispheres Induced by Complex Magnetic Fields. Journal of Nervous and Mental Diseases, 190, 533-541. http://dx.doi.org/10.1097/00005053-200208000-00006

[12] Newberg, A.B. (2010) Principles of Neurotheology. Ashgate, Burlington (VT).

[13] Joseph, R. (1982) The Neuropsychology of Development: Hemispheric Laterality, Limbic Language and the Origin of Thought. Journal of Clinical Psychology: Special Monograph Supplement, 38, 4-33. http://dx.doi.org/10.1002/1097-4679(198201)38:1<4::AID-JCLP2270380102>3.0.CO;2-J

[14] Durkheim, E. (1951). Le Suicide: Etude de Sociologie. Routledge, New York.

[15] Jackson, J.H. and Colman, W.S. (1898) Case of Epilepsy with Tasting Movements and "Dreamy State”. Brain, 21, 580-590. http://dx.doi.org/10.1093/brain/21.4.580

[16] Kuijsten, M. (2006) Reflections of the Dawn of Consciousness: Julian Jaynes’s Bicameral Mind Theory Revisited. Julian Jaynes Society, NV.

[17] Suedfeld, P. and Mocellin, J.S.P. (1987) The "Sensed Presence" in Unusual Environments. Environment and Behavior, 19, 33-52. http://dx.doi.org/10.1177/0013916587191002 
[18] Persinger, M.A., Tiller, S.G. and Koren, S.A. (2000) Experimental Simulation of Haunt Experience and Elicitation of Paroxysmal Electroencephalographic Activity by Transcrebral Magnetic Field: Induction of a Synthetic Ghost? Perceptual and Motor Skills, 90, 659-674. http://dx.doi.org/10.2466/pms.2000.90.2.659

[19] George, M.S. and Bohning, D.E. (2002) Measuring Brain Connectivity with Functional Imaging and Transcranial Magnetic Stimulation. In: Davis, K.L., Charney, D., Coyle, J.T. and Nemeroff, C., Eds., Neuropsychopharmacology: The 5th Generation of Progress, Lippincott Williams \& Wilkins, Philadelphia, 392-410.

[20] Koren, S.A., Bosage, W.E. and Persinger, M.A. (2015) Magnetic Fields Generated by Optical Coupler Circuits May Also Be Containment Loci for Entanglement of P-N Junction-Plasma Cell Membrane Photons within Exposed Living Systems. International Letters of Chemistry, Physics and Astronomy, 3, 84-105.

[21] Ruttan, L.A., Persinger, M.A. and Koren, S.A. (1990) Enhancement of Temporal-Lobe Related Experiences during Brief Exposures to Milligauss Intensity Extremely Low Frequency Magnetic Fields. Journal of Bioelectricity, 9, 33-54. http://dx.doi.org/10.3109/15368379009027758

[22] Cook, C.M. and Persinger, M.A. (2001) Geophysical Variables and Behavior: XCII. Experimental Elicitation of a Sentient Being by Right Hemispheric, Weak Magnetic Fields: Interactions with Temporal Lobe Sensitivity. Perceptual and Motor Skills, 92, 447-448. http://dx.doi.org/10.2466/pms.2001.92.2.447

[23] Persinger, M.A., Makarec, K. and Bradley, J.-C. (1988) Characteristics of Limbic Seizures Evoked by Peripheral Injections of Lithium and Pilocarpine. Physiology and Behavior, 44, 27-37. http://dx.doi.org/10.1016/0031-9384(88)90342-3

[24] Persinger, M.A. (1974) The Paranormal: The Patterns and Mechanisms. M. S. S. Information, New York.

[25] Persinger, M.A. (2003) The Sensed Presence Within Experimental Settings: Implications for the Male and Female Concept of Self. The Journal of Psychology, 137, 5-16. http://dx.doi.org/10.1080/00223980309600595

[26] Persinger, M.A. (2001) The Neuropsychiatry of Paranormal Experiences. Journal of Neuropsychiatry and Clinical Neuroscience, 13, 515-524. http://dx.doi.org/10.1176/jnp.13.4.515

[27] Persinger, M.A. (1987) MMPI Profiles of Normal People Who Display Frequent Temporal Lobe Signs. Perceptual and Motor Skills, 64, 1112-1114. http://dx.doi.org/10.2466/pms.1987.64.3c.1112

[28] Baker-Price, L. and Persinger, M.A. (2003) Intermittent Burst-Firing Weak (1 MicroTesla) Magnetic Fields Reduce Psychometric Depression in Patients Who Sustained Closed Head Injuries: A Replication and Electroencephalographic Validation. Perceptual and Motor Skills, 96, 964-974. http://dx.doi.org/10.2466/pms.2003.96.3.965

[29] Persinger, M.A., Hoang, V. and Baker-Price, L. (2009) Entrainment of Stage 2 Sleep Spindles by Weak Transcererbal Magnetic Stimulation of an “Epileptic” Woman. Electromagnetic Biology and Medicine, 28, 374-382. http://dx.doi.org/10.3109/15368370903206648

[30] Fleming, J.L., Persinger, M.A. and Koren, S.A. (1994) Magnetic Pulses Elevate Nociceptive Thresholds: Comparisons with Opiate Receptor Compounds in Normal and Seizure-Induced Brain-Damaged Rats. Electro-and Magnetobiology, 13, 67-75. http://dx.doi.org/10.3109/15368379409030699

[31] Martin, L.J., Koren, S.A. and Persinger, M.A. (2004) Thermal Analgesic Effects from Weak, Complex Magnetic Fields and Pharmacological Interactions. Pharmacology, Biochemistry and Behavior, 78, 217-227. http://dx.doi.org/10.1016/j.pbb.2004.03.016

[32] Martin, L.J. and Persinger, M.A. (2005) The Influence of Various Pharmacological Agents on the Analgesia Induced by Applied Complex Magnetic Field Treatment: A Receptor Potpourri. Electromagnetic Biology and Medicine, 7, 87-97. http://dx.doi.org/10.1080/15368370500205431

[33] Martin, L.J. and Persinger, M.A. (2005) Thermal Analgesic Effects from Weak (1 microT), Complex Magnetic Fields: Critical Parameters. Electromagnetic Biology and Medicine, 7, 411-418.

[34] Aaen-Stockdale, C. (2012) Neuroscience for the Soul. The Psychologist, 25, 520-523.

[35] Booth, J.N. and Persinger, M.A. (2009) Discrete Shifts within the Theta Band between the Frontal and Parietal Regions of the Right Hemisphere and Experiences of a Sensed Presence. Journal of Neuropsychiatry and Clinical Neuroscience, 21, 279-283. http://dx.doi.org/10.1176/jnp.2009.21.3.279

[36] Dewhurst, K. and Beard, A.W. (1970) Sudden Religious Conversions in Temporal Lobe Epilepsy. British Journal of Psychiatry, 117, 497-507. http://dx.doi.org/10.1192/bjp.117.540.497

[37] Slater, E. and Beard, A.W. (1963) Schizophrenia-Like Psychosis of Epilepsy: British Journal of Psychiatry, 109, 95-150. http://dx.doi.org/10.1192/bjp.109.458.95

[38] Bear, D.M. (1979) Temporal Lobe Epilepsy—A Syndrome of Sensory-Limbic Hyperconnection. Cortex, 15, 357-384. http://dx.doi.org/10.1016/S0010-9452(79)80064-7

[39] Persinger, M.A. and Tiller, S.G. (2008) Case Report: A Prototypical Spontaneous "Sensed Presence” of a Sentient Being and Concomitant Electroencephalographic Activity in the Clinical Laboratory. Neurocase, 14, 425-430. 
http://dx.doi.org/10.1080/13554790802406172

[40] Persinger, M.A. (1984) Striking EEG Profiles from Single Episodes of Glossolalia and Transcendental Meditation. Perceptual and Motor Skills, 58, 127-133. http://dx.doi.org/10.2466/pms.1984.58.1.127

[41] Kubie, L.S. (1953) Some Implications for Psychoanalysis of Modern Concepts of the Organization of the Brain. Psychoanalytic Quarterly, 22, 21-68.

[42] Williams, D. (1956) The Structure of Emotions Reflected in Epileptic Experiences. Brain, 79, 29-67. http://dx.doi.org/10.1093/brain/79.1.29

[43] Halgren, E., Walter, R.D., Cherlow, D.G. and Crandall, P.H. (1978) Mental Phenomena Evoked by Electrical Stimulation of the Human Hippocampal Formation and Amygdala. Brain, 101, 83-117. http://dx.doi.org/10.1093/brain/101.1.83

[44] Stevens, J.R., Mark, V.H., Erwin, F., Pacheco, P. and Suematsu, K. (1969) Deep Temporal Stimulation in Man: Long Latency, Long Lasting Changes. Archives of Neurology, 21, 157-169. http://dx.doi.org/10.1001/archneur.1969.00480140057006

[45] Bancaud, J., Brunet-Bourgin, F., Chauvel, P. and Halgren, E. (1994) Anatomical Origin of Déjà Vu and Vivid "Memories” in Human Temporal Lobe Epilepsy. Brain, 117, 71-90. http://dx.doi.org/10.1093/brain/117.1.71

[46] Gloor, P., Olivier, A., Quesney, L.F., Andermann, F. and Horowitz, S. (1982) The Role of the Limbic System in Experimental Phenomena of Temporal Lobe Epilepsy. Annals of Neurology, 12, 129-144. http://dx.doi.org/10.1002/ana.410120203

[47] Mahl, G.F., Rothenberg, A., Delgado, J.M.R. and Hamlin, H. (1964) Psychological Responses in the Human to Intracerebral Electrical Stimulation. Psychosomatic Medicine, 26, 337-368. http://dx.doi.org/10.1097/00006842-196407000-00005

[48] Picard, F. (2013) State of Belief, Subjective Certainty and Bliss as a Product of Cortical Dysfunction. Cortex, 49, 2494-2500. http://dx.doi.org/10.1016/j.cortex.2013.01.006

[49] Persinger, M.A. and Makarec, K. (1992) The Feeling of a Presence and Verbal Meaningfulness in Context of Temporal Lobe Function: Factor Analytic Verification of the Muses? Brain and Cognition, 20, 217-226. http://dx.doi.org/10.1016/0278-2626(92)90016-F

[50] Makarec, K. and Persinger, M.A. (1990) Electroencephalographic Validation of Temporal Lobe Signs Inventory in the Normal Population. Journal of Research in Personality, 24, 323-337. http://dx.doi.org/10.1016/0092-6566(90)90024-Z

[51] Munro, C. and Persinger, M.A. (1992) Relative Right Temporal-Lobe Theta Activity Correlates with Vingiano’s Hemispheric Quotient and the "Sensed Presence". Perceptual and Motor Skills, 75, 899-903.

[52] Urgesi, C., Aglioti, S.M., Skrap, M. and Fabbro, F. (2010) The Spiritual Brain: Selective Cortical Lesions Modulate Human Self-Transcendence. Neuron, 65, 309-319. http://dx.doi.org/10.1016/j.neuron.2010.01.026

[53] Taber, K.H. and Hurley, R.A. (2007) Neuroimaging in Schizophrenia: Misattributions and Religious Delusions. Journal of Neuropsychiatry and Clinical Neuroscience, 19, 1-4.

[54] Wuerful, J., Krishnamoorthy, E.S., Brown, R.J., Limeux, L., Koepp, M., Tebartz van Elst, L. and Trimble, M.R. (2004) Religiosity Is Associated with Hippocampal but Not Amygdala Volumes in Patients with Refractory Epilepsy. Journal Neurology, Neurosurgery and Psychiatry, 75, 640-642. http://dx.doi.org/10.1136/jnnp.2003.06973

[55] Owen, A.D., Hayward, R.D., Koenig, H.G., Steffens, D.C. and Payne, M.E. (2011) Religious Factors and Hippocampal Atrophy in Late Life. PLoS ONE, 6, e17006. http://dx.doi.org/10.1371/journal.pone.0017006

[56] Chan, D., Anderson, V., Pijnenburg, Y., Whitwell, J., Barnes, J., Scahill, R., Stevens, J.M., Barkhof, F., Schelten, P., Rossor, M.N. and Fox, N.C. (2009) The Clinical Profile of Right Temporal Atrophy. Brain, 132, 1287-1298. http://dx.doi.org/10.1093/brain/awp037

[57] Persinger, M.A. (1983) Religious and Mystical Experiences as Artifacts of Temporal Lobe Function: A General Hypothesis. Perceptual Motor Skills, 57, 1255-1262. http://dx.doi.org/10.2466/pms.1983.57.3f.1255

[58] Kanazawa, S. (2009) IQ and the Values of Nations. Journal of Biosocial Sciences, 41, 537-556. http://dx.doi.org/10.1017/S0021932009003368

[59] Fingelkurts, A.A. and Fingelkurts, A.A. (2009) Is Our Brain Hardwired to Produce God or Is Our Brain Hardwired to Perceive God? A Systematic Review on the Role of the Brain in Mediating Religious Experiences. Cognitive Processes, 10, 293-326. http://dx.doi.org/10.1007/s10339-009-0261-3

[60] Kanai, R., Feilden, T., Firth, C. and Rees, G. (2011) Political Orientations Are Correlated with Brain Structure in Young Adults. Current Biology, 21, 677-680. http://dx.doi.org/10.1016/j.cub.2011.03.017

[61] Goldensohn, E.S. (1983) Symptomatology of Nonconvulsive Seizures: Ictal and Postictal. Epilepsia, 24, S5-S21. http://dx.doi.org/10.1111/j.1528-1157.1983.tb04643.x

[62] Hines, M.E., Kubu, C.S., Roberts, R.J. and Varney, N.R. (1995) Characteristics and Mechanisms of the Epileptic 
Spectrum Disorder: an Exploratory Model. Applied Neuropsychology, 2, 1-6. http://dx.doi.org/10.1207/s15324826an0201_1

[63] Beauregard, M. and Paquette, V. (2006) Neural Correlates of a Mystical Experience in Carmelite Nuns. Neuroscience Letters, 405, 186-190. http://dx.doi.org/10.1016/j.neulet.2006.06.060

[64] Babb, T.L., Wilson, C.L. and Isokawa-Akesson, M. (1987) Firing Patterns of Human Limbic Neurons during Stereoencephalography (SEEG) and Clinical Temporal Lobe Seizures. Electroencephalography and Clinical Neurophysiology, 66, 467-482. http://dx.doi.org/10.1016/0013-4694(87)90093-9

[65] Bozzi, Y., Dunleavy, M. and Henshall, D.C. (2011) Cell Signaling Underlying Epileptic Behavior. Frontiers in Behavioral Neuroscience, 5, 45. http://dx.doi.org/10.3389/fnbeh.2011.00045

[66] Granqvist, P., Fredrikson, M., Unge, P., Hagenfeldt, A., Valind, S., Larhammar, D., Larsson, M., et al. (2005) Sensed Presence and Mystical Experiences Are Predicted by Suggestibility, Not Application of Transcranial Weak magnetic Fields. Neuroscience Letters, 379, 1-6. http://dx.doi.org/10.1016/j.neulet.2004.10.057

[67] Persinger, M.A. and Koren, S.A. (2005) A Response to Granqvist et al. "Sensed Presence and Mystical Experiments Are Predicted by Suggestibility, Not by Application of Transcranial Weak Magnetic Fields”. Neuroscience Letters, 308, 346-347. http://dx.doi.org/10.1016/j.neulet.2005.03.060

[68] French, C.C., Hague, U., Bunton-Stasyshyn, R. and Davis, R. (2009) The "Haunt” Project: An Attempt to Build a “Haunted” Room by Manipulating Complex Electromagnetic Fields and Infrasound. Cortex, 45, 619-629. http://dx.doi.org/10.1016/j.cortex.2007.10.011

[69] Tsang, E.W., Koren, S.A. and Persinger, M.A. (2004) Electrophysiological and Quantitative Electroencephalographic Measurements after Treatment with Transcerebral Magnetic Fields Generated by Compact Disc through a Computer Sound Card: The Shakti Treatment. International Journal of Neuroscience, 114, 1013-1024. http://dx.doi.org/10.1080/00207450490461323

[70] Gendle, M.H. and McGrath, M. (2012) Can the 8-Coil Shakti Alter Subjective Emotional Experience? A Randomized, Placebo-Controlled Study. Perceptual and Motor Skills, 114, 217-235. http://dx.doi.org/10.2466/02.24.PMS.114.1.217-235

[71] St-Pierre, L.S. and Persinger, M.A. (2006) Experimental Facilitation of the Sensed Presence Is Predicted by Specific Patterns of Applied Magnetic Fields Not by Suggestibility: Re-Analysis of 19 Experiments. International Journal of Neuroscience, 116, 1-18.

[72] Spiegel, H. and Spiegel, D. (1978) Trance and Treatment: Clinical Uses of Hypnosis. Basic Books, New York.

[73] Murugan, M.J. and Persinger, M.A. (2014) Comparisons of Responses by Planarians to MicroMolar to Attomolar Dosages of Morphine and Naloxone and/or Weak Pulsed Magnetic Fields: Revealing Receptor Subtype Affinities and Non-Specific Effects. International Journal of Radiation Biology, 90, 833-840.

[74] Freeman, J. and Persinger, M.A. (1996) Repeated Verbal Interruption during Exposure to Complex Transcerebral Magnetic Fields Elicit Irritability: Implications for Opiate Effects. Perceptual and Motor Skills, 82, 639-642. http://dx.doi.org/10.2466/pms.1996.82.2.639

[75] Thomas, A.W., Kavaliers, M., Prato, F.S. and Ossenkopp, K.-P. (1997) Pulsed Magnetic Field Induces “Analgesia” in the Land Snail and the Effects of Mu, Delta and Kappa Opioid Receptor Agonists/Antagonists. Peptides, 18, 703-709. http://dx.doi.org/10.1016/S0196-9781(97)00004-1

[76] Del Seppia, V., Ghione, S., Luschi, P., Ossenkopp, K.-P., Choleris, E. and Kavaliers, M. (2007) Pain Perception and Electromagnetic Fields. Neuroscience and Biobehavioral Reviews, 31, 619-642. http://dx.doi.org/10.1016/j.neubiorev.2007.01.003

[77] Tinoca, C.A. and Oritz, J.P.L. (2014) Magnetic Stimulation of the Temporal Cortex: A Partial “God Helmet” Replication Study. Journal of Consciousness Exploration \& Research, 5, 234-257.

[78] Richards, P.M., Persinger, M.A. and Koren, S.A. (1993) Modification of Activation and Evaluation Properties of Narratives by Weak Complex Patterned Magnetic Field Patterns That Simulate Limbic Burst Firing. International Journal of Neuroscience, 71, 71-85. http://dx.doi.org/10.3109/00207459309000594

[79] Saroka, K.S. and Persinger, M.A. (2013) Potential Production of Hughlings Jackson’s "Parasitic Consciousness” by Physiologically-Patterned Weak Transcerebral Magnetic Fields: QEEG and Source Localization. Epilepsy and Behavior, 28, 395-407. http://dx.doi.org/10.1016/j.yebeh.2013.05.023

[80] Persinger, M.A. and Saroka, K.S. (2013) Minimum Attenuation of Physiologically-Patterned, 1 MicroTesla Magnetic Fields through Simulated Skull and Cerebral Space. Journal of Electromagnetic Analysis and Applications, 5, 151-155. http://dx.doi.org/10.4236/jemaa.2013.54024

[81] Le Chapellier, P. and Matta, B. (2012) Cellular Psychology Assumption: Based Upon Exterior Algebra, It Contributes to Explaining the Variability of the Bioeffects of Magnetic and Electromagnetic Fields. Neuroscience \& Medicine, $\mathbf{3}$, 
251-269. http://dx.doi.org/10.4236/nm.2012.33029

[82] Buckner, C.A., Buckner, A.L., Koren, S.A., Persinger, M.A. and Lafrenie, R.M. (2014) Inhibition of Cancer Cell Growth by Exposure to a Specific Time-Varying Electromagnetic Field Involves T-Type Calcium Channels. PLoS ONE, 10, e0124136. http://dx.doi.org/10.1371/journal.pone.0124136

[83] Su, H., Sochivko, D., Becker, A., Chen, J., Jiang, Y., Yaari, Y. and Beck, H. (2002) Upregulation of a T-Type Ca ${ }^{2+}$ Channel Causes a Long-Lasting Modification of Neuronal Firing Mode after Status Epilepticus. The Journal of Neuroscience, 22, 3645-3655.

[84] Sutula, T., Cascino, G., Cavazos, J., Parada, I. and Ramirez, L. (1989) Moss Fiber Synaptic Reorganization in the Epileptic Human Temporal Lobe. Annals of Neurology, 26, 321-330. http://dx.doi.org/10.1002/ana.410260303

[85] Wolfart, J. and Roeper, J. (2002) Selective Coupling of T-Type Calcium Channels to SK Potassium Channels Prevents Intrinsic Bursting in Dopaminergic Midbrain Neurons. The Journal of Neuroscience, 22, 3404-3413.

[86] Persinger, M.A. (2010) $10^{-20}$ Joules as a Neuromolecular Quantum in Medicinal Chemistry: An Alternative Approach to Myriad Molecular Pathways. Current Medicinal Chemistry, 17, 3094-3098. http://dx.doi.org/10.2174/092986710791959701

[87] Persinger, M.A. (2014) Infrasound, Human Health, and Adaptation: An Integrative Overview of Recondite Hazards in a Complex Environment. Natural Hazards, 70, 501-525. http://dx.doi.org/10.1007/s11069-013-0827-3

[88] Persinger, M.A. (2014) Convergences of Numbers of Synapses and Quantum Foci within Human Brain Space: Quantitative Implications of the Photon as a Source of Cognition. International Letters of Chemistry, Physics and Astronomy, 11, 59-66. http://dx.doi.org/10.18052/www.scipress.com/ILCPA.30.59

[89] Persinger, M.A. and St-Pierre, L.S. (2015) The Physical Bases to Consciousness: Implications of Convergent Quantifications. Journal of Systems and Integrative Neuroscience, 1, 55-64.

[90] Persinger, M.A. and Saroka, K.S. (2014) Comparable Proportions of Classes of Experiences and Intracerebral Consequences for Surgical Stimulation and External Application of Weak Magnetic Fields Patterns: Implications for Converging Effects in Complex Partial Seizures. Epilepsy and Behavior, 27, 220-224. http://dx.doi.org/10.18052/www.scipress.com/ILCPA.30.59

[91] Persinger, M.A. (1987) Neuropsychological Bases of God Beliefs. Praeger, New York.

[92] Murphy, T. (2015) Sacred Pathways: The Brain’s Role in Religion and Mystical Experiences. 2nd Edition, Create Space Publishers, North Charleston. 